\title{
Allgemeines Literatur- und Quellenverzeichnis
}

\section{Textsammlungen}

- Amstutz/Pichonnaz/Probst/Werro, Droit privé européen. Directives choisies, 2. Aufl., Stämpfli, Bern 2011

- Artz/Staudinger, Europäisches Verfahrens-, Kollisions- und Privatrecht, C.F. Müller, Heidelberg 2010

- Grolimund/Mosimann, Internationales Privat- und Zivilverfahrensrecht der Europäischen Union, 2. Aufl., Dike-Verlag, Zürich/St. Gallen 2015

- Grundmann/Riesenhuber, Textsammlung Europäisches Privatrecht. Vertrags- und Schuldrecht, Arbeitsrecht, Gesellschaftsrecht, 3. Aufl., De Gruyter, Berlin/New York 2020

- Jayme/Hausmann, Internationales Privat- und Verfahrensrecht - Textausgabe, 20. Aufl., C.H. Beck, München 2020

- Pechstein/Domröse, Europarecht - Textsammlung, 3. Aufl., Mohr Siebeck, Tübingen 2018

- Schulze/Zimmermann, Europäisches Privatrecht - Basistexte, 6. Aufl., Nomos-Verlag, Baden-Baden 2020

\section{Wissenschaftliche Entwürfe}

- von Bar/Clive/Schulte-Nölke (Hrsg.), Principles, Definitions and Model Rules of European Private Law. Draft Common Frame of Reference (DCFR), Full Edition, Sellier, München 2009

- Beale/Lando, The Principles of European Contract Law, Part I, Performance, Non Performance and Remedies, Prepared by the Commission on European Contract Law, Dordrecht 1995

- von Bar/Zimmermann (Hrsg. und Übersetzer), Grundregeln des Europäischen Vertragsrechts, Teile I und II, München 2002

- von Bar/Zimmermann (Hrsg. und Übersetzer), Grundregeln des Europäischen Vertragsrechts, Teil III, München 2005

- Gandolfi (Hrsg.), Code Européen des Contrats, Avant-projet, Livre Premier, Mailand 2001

- Jansen/Zimmermann (Hrsg.), Commentaries on European Contract Laws, Oxford University Press, Oxford 2018

- Lando/Beale (Hrsg.), Principles of European Contract Law, Parts I and II, Den Haag 2000

- Lando/Clive/Prüm/Zimmermann (Hrsg.), Principles of European Contract Law, Part III, Den Haag 2003

\section{Fall- und Entscheidungssammlungen; Übungsbücher}

- Brödermann/Rosengarten, Internationales Privat- und Zivilverfahrensrecht (IPR/IZVR). Anleitung zur systematischen Fallbearbeitung, 8. Aufl., Vahlen, München 2019

- Coester-Waltjen/Mäsch, Übungen in internationalem Privatrecht und Rechtsvergleichung, 5. Aufl., De Gruyter, Berlin/New York 2017

- Franck/Möslein, Fälle zum Europäischen Privat- und Wirtschaftsrecht, C.H. Beck, München 2005

- Fuchs/Hau/Thorn, Fälle zum Internationalen Privatrecht, 5. Aufl. 2019

- Hartkamp/Sieburgh/Devroe, Cases, Materials and Text on European Law and Private Law, Hart, Oxford 2017 
- Kadner Graziano, Europäisches Vertragsrecht: Übungen zur Rechtsvergleichung und Harmonisierung des Rechts, Nomos-Verlag, Baden-Baden, 2008

- Rauscher, Klausurenkurs im Internationalen Privatrecht, 4. Aufl. 2019

- Rösler, Internationales Privatrecht (Reihe Prüfe dein Wissen), 6. Aufl. 2020

- Schulze/Engel/Jones, Casebook Europäisches Privatrecht, Nomos-Verlag, Baden-Baden 2000

\section{Lehrbücher, Einführungen}

\section{a) Zum Europäischen Privatrecht}

- $\quad$ Heiderhoff, Europäisches Privatrecht, 5. Aufl., C.F. Müller, Heidelberg 2020

- Herresthal, § 2: Vertragsrecht, in: Langenbucher (Hrsg.), Europäisches Privat- und Wirtschaftsrecht, 4. Aufl., Nomos-Verlag, Baden-Baden 2017

- Langenbucher, § 1: Europarechtliche Methodenlehre, in: Langenbucher (Hrsg.), Europäisches Privat- und Wirtschaftsrecht, 4. Aufl., Nomos-Verlag, Baden-Baden 2017

- Ranieri, Europäisches Obligationenrecht - Ein Handbuch mit Texten und Materialien, 3. Aufl., Springer, Wien 2009

- $\quad$ Riesenhuber, EU-Vertragsrecht, Mohr Siebeck, Tübingen 2013

- Schulze/Zoll, Europäisches Vertragsrecht, 2. Aufl., Nomos-Verlag, Baden-Baden 2017

- Schulze/Zoll, European Contract Law, 2. Aufl., C.H. Beck/Hart/Nomos, Baden-Baden 2018

- Weatherill, Contract Law of the Internal Market, Intersentia, Antwerpen 2016

\section{b) Zum Europäischen IPR}

- Bach/Huber, Internationales Privat- und Prozessrecht, C.H. Beck, München 2020

- von Bar/Mankowski, Internationales Privatrecht, Band 2: Besonderer Teil, 2. Aufl., C. H. Beck, München 2019

- van Calster, European Private International Law, 3. Aufl., Hart, Oxford/Portland 2021

- von Hoffmann/Thorn, Internationales Privatrecht, 9. Aufl., C.H. Beck, München 2007

- Hüßtege/Ganz, Internationales Privatrecht einschließlich Grundzüge des Internationalen Verfahrensrechts, 5. Aufl., C.H. Beck, München 2013

- Junker, Internationales Privatrecht, 4. Aufl., C.H. Beck, München 2021

- Kienle, Internationales Privatrecht, 2. Aufl., Verlag Franz Vahlen, München 2010

- Koch/Magnus/Winkler von Mohrenfels, IPR und Rechtsvergleichung, 4. Aufl., C.H. Beck, München 2010

- Köhler, Examinatorium Internationales Privatrecht, 2. Aufl., Nomos-Verlag, Baden-Baden 2020

- Rauscher, Internationales Privatrecht. Mit internationalem Verfahrensrecht, 5. Aufl., C.F. Müller, Heidelberg 2017

- Rogerson, Collier's Conflict of Laws, 4. Aufl., Cambridge University Press, Cambridge 2013

- Stone on Private International Law in the European Union, 4. Aufl., Edward Elgar Publishing, Cheltenham 2018

- Stürner, § 8: Internationales Privatrecht, in: Langenbucher (Hrsg.), Europäisches Privat- und Wirtschaftsrecht, 4. Aufl., Nomos-Verlag, Baden-Baden 2017

- $\quad$ Torremans (Hrsg.), Cheshire, North \& Fawcett, Private International Law, 15. Aufl., Oxford University Press, Oxford 2017 


\section{Monographien, Handbücher, Sammelbände}

\section{a) Zum Europäischen Privatrecht}

- Alpa/Andenæs, Grundlagen des Europäischen Privatrechts, Springer, 2010

- Andenæs/Baasch Andersen, Theory and Practice of Harmonization, Edward Elgar Publishing, Cheltenham 2012

- Arnold (Hrsg.), Grundlagen eines europäischen Vertragsrechts, Sellier, München 2014

- Baldauf, Richtlinienverstoß und Verschiebung der Contra-legem-Grenze im Privatrechtsverhältnis. Der Konflikt zwischen Richtlinie und nationalem Recht bei der Rechtsanwendung, Tübingen 2013

- Baldus/Müller-Graff(Hrsg.), Die Generalklausel im Europäischen Privatrecht. Zur Leistungsfähigkeit der deutschen Wissenschaft aus romanischer Perspektive, München 2006

- Basedow/Hopt/Zimmermann (Hrsg.) Handwörterbuch des Europäischen Privatrechts (2 Bände), Mohr Siebeck, Tübingen 2009 (Studienausgabe 2011)

- Behme e.a. (Hrsg.), Perspektiven einer europäischen Privatrechtswissenschaft, JbJZRWiss 2016, Nomos-Verlag, Baden-Baden 2017

- Beil, Personale Differenzierung im Kaufrecht. Rechtsvergleichende Studie unter Einbeziehung deutscher und französischer Regelungen und internationaler Regelwerke (CISG, UNIDROIT PICC, (ESL, CFR), Mohr Siebeck, Tübingen 2018

- Bischoff, Die Europäische Gemeinschaft und die Konventionen des einheitlichen Privatrechts, Mohr Siebeck, Tübingen 2010

- Bron, Rechtsangleichung des Privatrechts auf Ebene der Europäischen Union, Nomos-Verlag, Baden-Baden 2011

- Cauffman/Smits (Hrsg.), The Citizen in European Private Law: Norm-setting, Enforcement and Choice, Intersentia, Antwerpen 2016

- Collins (Hrsg.), The EU Charter of Fundamental Rights and European Contract Law, Intersentia, Antwerpen 2017

- Dannemann/Vogenauer (Hrsg.), The Common European Sales Law in Context: Interactions with English and German Law, Oxford University Press, Oxford 2013

- Dastis, Das Rücktrittsrecht des Käufers im Europäischen Privatrecht, Nomos-Verlag, Baden-Baden 2017

- De Cristofaro/de Franceschi (Hrsg.), Consumer Sales Law in Europe - After the Implementation of the Consumer Sales Directive, Intersentia, Antwerpen 2016

- Drexler, Die richtlinienkonforme Interpretation in Deutschland und Frankreich, Nomos-Verlag, Baden-Baden 2012

- Ebers, Rechte, Rechtsbehelfe und Sanktionen im Unionsprivatrecht, Mohr Siebeck, Tübingen 2016

- Eidenmüller/Faust/Grigoleit/Jansen/Wagner/Zimmermann, Revision des Verbraucher-acquis, Mohr Siebeck, Tübingen 2011

- Franzen, Privatrechtsangleichung durch die Europäische Gemeinschaft, De Gruyter, Berlin/ New York 1999

- Fries, Verbraucherrechtsdurchsetzung, Mohr Siebeck, Tübingen 2016

- Gebauer, Grundfragen der Europäisierung des Privatrechts. Eine Untersuchung nationaler Ansätze unter Berücksichtigung des italienischen und des deutschen Rechts, Heidelberg 1998

- Gebauer/Teichmann (Hrsg.), Enzyklopädie des Europarechts Band 6: Europäisches Privat- und Unternehmensrecht, Nomos-Verlag, Baden-Baden 2016 
- Gebauer/Wiedmann (Hrsg.), Zivilrecht unter europäischem Einfluss. Die richtlinienkonforme Auslegung des BGB und anderer Gesetze, Kommentierung der wichtigsten EU-Verordnungen, 2. Aufl., Boorberg-Verlag, Stuttgart 2010

- Goanţă, Convergence in European Consumer Sales Law. A Comparative and Numerical Approach, Intersentia, Antwerpen 2016

- Grundmann, Europäisches Schuldvertragsrecht - das Europäische Recht der Unternehmensgeschäfte (nebst Texten und Materialien zur Rechtsangleichung), De Gruyter, Berlin/New York 1999

- Grundmann (Hrsg.), Systembildung und Systemlücken in Kerngebieten des Europäischen Privatrechts - Gesellschaftsrecht, Arbeitsrecht, Schuldvertragsrecht, Mohr Siebeck, Tübingen 2000

- Grundmann (Hrsg.), European Contract Law in the Digital Age, Intersentia, Antwerpen 2018

- Grundmann/Bianca (Hrsg.), EU Kaufrechts-Richtlinie - Kommentar, Otto Schmidt, Köln 2002

- Gsell/Herresthal (Hrsg.), Vollharmonisierung im Privatrecht - Die Konzeption der Richtlinie am Scheideweg?, Mohr Siebeck, Tübingen 2010

- Gutman, The Constitutional Foundations of European Contract Law: A Comparative Analysis, Oxford University Press, Oxford 2014

- Hartkamp, European Law and National Private Law, 2. Aufl., Intersentia, Antwerpen 2016

- Heiderhoff/Lohsse/Schulze (Hrsg.), EU-Grundrechte und Privatrecht, Nomos-Verlag, Baden-Baden 2016

- Heinze, Schadensersatz im Unionsprivatrecht. Eine Studie zu Effektivität und Durchsetzung des Europäischen Privatrechts am Beispiel des Haftungsrechts, Mohr Siebeck, Tübingen 2017

- Helleringer/Purnhagen (Hrsg.), Towards a European Legal Culture, C.H. Beck/Hart/Nomos, Baden-Baden 2014

- Henke, Enthält die Liste des Anhangs der Klauselrichtlinie 93/13/EWG Grundregeln des Europäischen Vertragsrechts?, Mohr Siebeck, Tübingen 2010

- Henninger, Europäisches Privatrecht und Methode. Entwurf einer rechtsvergleichend gewonnenen juristischen Methodenlehre, Mohr Siebeck, Tübingen 2009

- Herresthal, Rechtsfortbildung im europarechtlichen Bezugsrahmen, Methoden, Kompetenzen, Grenzen; dargestellt am Beispiel des Privatrechts, C. H. Beck, München 2006

- Höpfner, Die systemkonforme Auslegung: Zur Auflösung einfachgesetzlicher, verfassungsrechtlicher und europarechtlicher Widersprüche im Recht, Mohr Siebeck, Tübingen 2008

- Jansen, Binnenmarkt, Privatrecht und Europäische Identität. Eine historische und methodische Bestandsaufnahme, Mohr Siebeck, Tübingen 2004

- Jud/Wendehorst, (Hrsg.), Neuordnung des Verbraucherprivatrechts in Europa? Zum Vorschlag einer Richtlinie über Rechte der Verbraucher, Wien 2009

- Leible, Wege zu einem europäischen Privatrecht, Habilitationsschrift Bayreuth 2001

- Letto-Vanamo/Smits, (Hrsg.), Coherence and Fragmentation in European Private Law, München 2012

- van Leeuwen, European Standardisation of Services and its Impact on Private Law. Paradoxes of Convergence, Hart, Oxford 2017

- Lein, Die Verzögerung der Leistung im europäischen Vertragsrecht, Mohr Siebeck, Tübingen 2015

- $\quad$ von Leuken, Private Law and the Internal Market. Direct Horizontal Effect of the Treaty Provisions on Free Movement, Intersentia, Antwerpen 2017

- Lippstreu, Wege der Rechtsangleichung im Vertragsrecht. Vollharmonisierung, Mindestharmonisierung, optionales Instrument, Tübingen 2014

- Lohse, Rechtsangleichungsprozesse in der Europäischen Union. Instrumente, Funktionsmechanismen und Wirkparameter effektiver Harmonisierung, Mohr Siebeck, Tübingen 2017

- Lurger, Grundfragen der Vereinheitlichung des Vertragsrechts in der Europäischen Union, Springer, Wien 2002 
- Lüttringhaus, Vertragsfreiheit und ihre Materialisierung im Europäischen Binnenmarkt - Die Verbürgung und Materialisierung unionaler Vertragsfreiheit im Zusammenspiel von EU-Privatrecht, BGB und ZPO, Mohr Siebeck, Tübingen 2018

- Martens, Methodenlehre des Unionsrechts, Mohr Siebeck, Tübingen 2013

- Metzger, Extra legem, intra ius. Allgemeine Rechtsgrundsätze im europäischen Privatrecht, Mohr Siebeck, Tübingen 2009

- Micklitz/Sieburgh (Hrsg.), Primary EU Law and Private Law Concepts, Intersentia, Antwerpen 2017

- $\quad$ Miller, The Emergence of EU Contract Law - Exploring Europeanization, Oxford 2011

- Mittwoch, Vollharmonisierung und Europäisches Privatrecht - Methode, Implikationen und Durchführung, Berlin 2013

- Mörsdorf, Ungleichbehandlung als Norm. Eine dogmatische Analyse des unional determinierten Antidiskriminierungsrechts in Deutschland, Mohr Siebeck, Tübingen 2018

- Müller-Graff(Hrsg.), Gemeinsames Privatrecht in der Europäischen Gemeinschaft, Nomos-Verlag, 2. Aufl., Nomos-Verlag, Baden-Baden 1999

- Niglia, The Struggle for European Private Law. A Critique of Codification, Hart, Oxford 2017

- Perner, Grundfreiheiten, Grundrechte-Charta und Privatrecht, Mohr Siebeck, Tübingen 2013

- $\quad$ Reich, General Principles of EU Civil Law, Intersentia, Antwerpen 2013

- Remien, Zwingendes Vertragsrecht und Grundfreiheiten des EG-Vertrags, Mohr Siebeck, Tübingen 2003

- $\quad$ Riehm, Der Grundsatz der Naturalerfüllung, Mohr Siebeck, Tübingen 2015

- Riesenhuber (Hrsg.), Europäische Methodenlehre. Grundfragen der Methoden des Europäischen Privatrechts, 3. Aufl., De Gruyter, Berlin 2015

- Riesenhuber, System und Prinzipien des Europäischen Vertragsrechts, Berlin 2003

- Rosentritt, Die Gefahrtragung im europäischen und internationalen Kaufrecht. CISG, INCO-Terms, Vorschlag für ein Gemeinsames Europäisches Kaufrecht, Verbraucherrechterichtlinie und deutsches Recht in vergleichender Perspektive, Mohr Siebeck, Tübingen 2018

- Rybarz, Billigkeitserwägungen im Kontext des Europäischen Privatrechts, Mohr Siebeck, Tübingen 2011

- van Schagen, The Development of European Private Law in a Multilevel Legal Order, Intersentia, Antwerpen 2016

- Schmidt, Der Vertragsschluss. Ein Vergleich zwischen dem deutschen, französischen, englischen Recht und dem CESL, Mohr Siebeck, Tübingen 2013

- Schmidt-Kessel (Hrsg.), Der Gemeinsame Referenzrahmen, Entstehung, Inhalte, Anwendung, Sellier, München 2009

- Schmidt-Kessel (Hrsg.), Ein einheitliches europäisches Kaufrecht? Eine Analyse des Vorschlags der Kommission, Sellier, München 2012

- Schröder, Der Unternehmerregress beim Verbrauchsgüterkauf im Falle von grenzüberschreitenden Handelskäufen. Eine rechtsvergleichende und kollisionsrechtliche Betrachtung unter Berücksichtigung des deutschen und österreichischen Rechts sowie des UN-Kaufrechts, des DCFR und des Vorschlags für ein GEK, Mohr Siebeck, Tübingen 2017

- Schulte-Nölke/Zoll/Jansen/Schulze (Hrsg.), Der Entwurf für ein optionales europäisches Kaufrecht, Sellier, München 2012

- Schulze/Staudenmayer (Hrsg.), Digital Revolution: Challenges for Contract Law in Practice, Nomos-Verlag, Baden-Baden 2016

- Schulze/Staudenmayer (Hrsg.), EU Digital Law: Article-by-Article Commentary, Nomos-Verlag, Baden-Baden 2020

- Schulze/von Bar/Schulte-Nölke (Hrsg.), Der akademische Entwurf für einen Gemeinsamen Referenzrahmen, Mohr Siebeck, Tübingen 2008

- Sonnentag, Das Rückgewährschuldverhältnis, Mohr Siebeck, Tübingen 2016 
- Sponholz, Die unionsrechtlichen Vorgaben zu den Rechtsfolgen von Diskriminierungen im Privatrechtsverkehr, Nomos-Verlag, Baden-Baden 2017

- Starke, EU-Grundrechte und Vertragsrecht, Mohr Siebeck, Tübingen 2016

- Stempel, Treu und Glauben im Unionsprivatrecht, Mohr Siebeck, Tübingen 2016

- Stürner, Der Grundsatz der Verhältnismäßigkeit im Vertragsrecht, Mohr Siebeck, Tübingen 2010

- Stürner (Hrsg.), Vollharmonisierung im europäischen Verbraucherrecht?, München 2010

- Tamm, Verbraucherschutzrecht. Europäisierung und Materialisierung des deutschen Zivilrechts und die Herausbildung eines Verbraucherschutzprinzips, Mohr Siebeck, Tübingen 2011

- Twigg-Flesner (Hrsg.), Research Handbook on EU Consumer and Contract Law, 2016

- Twigg-Flesner (Hrsg.), The Cambridge Companion to European Union Private Law, Cambridge University Press, Cambridge 2010

- Wagner, Der Einfluss Europas auf das BGB. Gesetzgebungstechnik europarechtlich veranlasster Änderungsgesetze, Duncker\&Humblot, Berlin 2017

- Zoppel, Europäische Diskriminierungsverbote und Privatrecht, Mohr Siebeck, Tübingen 2015

\section{b) Zum Europäischen IPR}

- Arnold (Hrsg.), Grundfragen des Europäischen Kollisionsrechts, Mohr Siebeck, Tübingen 2016

- Basedow/Rühl/Ferrari/de Miguel Asensio (Hrsg.), Encyclopedia of Private International Law, Edward Elgar, Cheltenham 2017

- Baur/Mansel (Hrsg.), Systemwechsel im Europäischen Kollisionsrecht, C. H. Beck, München 2002

- Bernitt, Die Anknüpfung von Vorfragen im europäischen Kollisionsrecht, Mohr Siebeck, Tübingen 2010

- Bruinier, Der Einfluss der Grundfreiheiten auf das Internationale Privatrecht, Peter Lang, Frankfurt a.M. 2003

- Calliess/Renner (Hrsg.), Rome Regulations - Commentary, 3. Aufl., Wolters Kluwer, Alphen aan den Rijn 2020

- Fallon/Lagarde/Poillot-Peruzzetto, Quelle architecture pour un code européen de droit international privé?, Peter Lang, Frankfurt a. M. 2011

- Guinchard (Hrsg.), Rome I and Rome II in Practice, Intersentia, Antwerpen 2020

- Hauser, Eingriffsnormen in der Rom I-Verordnung, Mohr Siebeck, Tübingen 2012

- von Hein/Rühl (Hrsg.), Kohärenz im Internationalen Privat- und Verfahrensrecht der Europäischen Union, Mohr Siebeck, Tübingen 2016

- von Hein/Kieninger/Rühl (Hrsg.), How European is European Private International Law? Sources, Court Practice, Academic Discourse, Intersentia, Antwerpen 2019

- Hemler, Die Methodik der „Eingriffsnorm“ im modernen Kollisionsrecht - zugleich ein Beitrag zum Internationalen Öffentlichen Recht und zur Natur des ordre public, Mohr Siebeck, Tübingen 2019

- Köhler, Eingriffsnormen - Der »unfertige Teil« des europäischen IPR, Mohr Siebeck, Tübingen 2013

- Kroll-Ludwigs, Die Rolle der Parteiautonomie im europäischen Kollisionsrecht, Mohr Siebeck, Tübingen 2013

- Leible/Unberath (Hrsg.), Brauchen wir eine Rom 0-Verordnung?, Jenaer Wissenschaftliche Verlagsgesellschaft, Jena 2013

- Leifeld, Das Anerkennungsprinzip im Kollisionsrechtssystem des internationalen Privatrechts, Mohr Siebeck, Tübingen 2010 
- Lüttringhaus, Grenzüberschreitender Diskriminierungsschutz - Das internationale Privatrecht der Antidiskriminierung, Mohr Siebeck, Tübingen 2010

- Nehne, Methodik und allgemeine Lehren des europäischen Internationalen Privatrechts, Mohr Siebeck, Tübingen 2012

- Nietner, Internationaler Entscheidungseinklang im europäischen Kollisionsrecht, Mohr Siebeck, Tübingen 2016

- $\quad$ Rentsch, Der gewöhnliche Aufenthalt im System des Europäischen Kollisionsrechts, Mohr Siebeck, Tübingen 2017

- Repasi, Wirkungsweise des unionsrechtlichen Anwendungsvorrangs im autonomen IPR, Mohr Siebeck, Tübingen 2018

- $\quad$ Rühl, Statut und Effizienz. Ökonomische Grundlagen des Internationalen Privatrechts, Mohr Siebeck, Tübingen 2011

- Schilling, Binnenmarktkollisionsrecht, De Gruyter, Berlin 2006

- Schwemmer, Anknüpfungsprinzipien im europäischen Kollisionsrecht. Integrationspolitische Zielsetzungen und das Prinzip der engsten Verbindung, Mohr Siebeck, Tübingen 2018

- Stone/Farah (Hrsg.), Research Handbook on EU Private International Law, Edward Elgar, Cheltenham 2015

- Thoma, Die Europäisierung und die Vergemeinschaftung des nationalen ordre public, Mohr Siebeck, Tübingen 2007

- $\quad$ Trüten, Die Entwicklungen des Internationalen Privatrechts in der Europäischen Union. Auf dem Weg zu einem europäischen IPR-Gesetz, Nomos/Stämpfli, Baden-Baden/Basel 2015

- Weller (Hrsg.), Europäisches Kollisionsrecht, Nomos-Verlag, Baden-Baden 2016

- Wilke, A Conceptual Analysis of European Private International Law. The General Issues in the EU and its Member States, Intersentia, Antwerpen 2019

\section{Zeitschriften}

- Common Market Law Review CML Rev.

- Contratto e impresa / Europa

- $\quad$ Europäisches Wirtschafts- und Steuerrecht (EWR)

- $\quad$ European Review of Contract Law (ERCL)

- $\quad$ European Review of Private Law (ERPL)

- Europäische Zeitschrift für Wirtschaftsrecht (EuZW)

- Praxis des Internationalen Privat- und Verfahrensrechts (IPRax)

- Journal of European Consumer and Market Law (EuCML) (früher: Zeitschrift für Europäisches Unternehmens- und Verbraucherrecht, euvr)

- Journal of Private International Law (JPIL)

- $\quad$ Rabels Zeitschrift für ausländisches und internationales Privatrecht (RabelsZ)

- Revue des contrats

- Yearbook of Private International Law

- Zeitschrift für das Privatrecht der Europäischen Union (GPR) (früher: Zeitschrift für Gemeinschaftsprivatrecht)

- Zeitschrift für Europäisches Privatrecht (ZEuP) 


\title{
7. Rechtsprechungsübersichten
}

\author{
a) Zum Europäischen Privatrecht
}

\author{
aa) In der ZEuP
}

Kohler/Seyr/Puffer-Mariette, ZEuP 2020, 366 (zur Rspr. des EuGH im Jahre 2018); Kohler/Seyr/PufferMariette, ZEuP 2019, 126 (zur Rspr. des EuGH im Jahre 2017); Kohler/Seyr/Puffer-Mariette, ZEuP 2018, 177 (zur Rspr. des EuGH im Jahre 2016); Kohler/Seyr/Maderbacher, ZEuP 2017, 431 (zur Rspr. des EuGH im Jahre 2015); Kohler/Puffer-Mariette/Maderbacher, ZEuP 2016, 464 (zur Rspr. des EuGH im Jahre 2014); Kohler/Seyr/Puffer-Mariette, ZEuP 2015, 335 (zur Rspr. des EuGH im Jahre 2013); Kohler/Puffer-Mariette, ZEuP 2014, 696 (EuGH und Privatrecht, ein Rückblick nach 60 Jahren); Kohler/Seyr/Puffer-Mariette, ZEuP 2014, 116 (zur Rspr. des EuGH im Jahre 2012); Kohler/Seyr/Puffer-Mariette, ZEuP 2013, 323 (zur Rspr. des EuGH im Jahre 2011); Kohler/Seyr/ Puffer-Mariette, ZEuP 2011, 874 (zur Rspr. des EuGH im Jahre 2010); Kohler/Seyr/Puffer-Mariette, ZEuP 2011, 145 (zur Rspr. des EuGH im Jahre 2009); Kohler/Knapp, ZEuP 2010, 620 (zur Rspr. des EuGH im Jahre 2008); Kohler/Denkinger/Seyr, ZEuP 2009, 322 (zur Rspr. des EuGH im Jahre 2007); Kohler/Knapp, ZEuP 2007, 484 (zur Rspr. des EuGH im Jahre 2005); Kohler/Knapp, ZEuP 2004, 705 (zur Rspr. des EuGH im Jahre 2002).

\section{bb) In der EWS}

Kas/Micklitz, EWS 2018, 181 und 241 (Rechtsprechungsübersicht zum Europäischen Vertrags- und Deliktsrecht, 2014-2018); Kas/Micklitz, EWS 2013, 314 und 353 (Rechtsprechungsübersicht zum Europäischen Vertrags- und Deliktsrecht, 2008-2013); Micklitz, EWS 2008, 353 (Rechtsprechung zum Europäischen Verbraucherrecht - Vertrags- und Deliktsrecht, Aufbereitung der Rechtssachen im Anschluss an EWS 2006, 1); Micklitz, EWS 2006, 1 (Rechtsprechungsübersicht zum Europäischen Verbraucherrecht: Vertrags- und Deliktsrecht).

\section{cc) In der GPR}

- Länderberichte England von Twigg-Flesner, GPR 2003/04, 249; GPR 2003/04, 123

- Länderberichte Frankreich von Klötgen/Mansuy, GPR 2020, 158 (Klötgen); GPR 2020, 2; GPR 2019, 154; GPR 2019, 2; GPR 2018, 130; GPR 2018, 2; GPR 2017, 106; GPR 2016, 271; GPR 2015, 164; GPR 2014, 249; GPR 2014, 80; GPR 2013, 259; GPR 2013, 79; GPR 2012, 242; GPR 2012, 70; GPR 2011, 247; GPR 2011, 69; GPR 2010, 218; GPR 2010, 65; GPR 2008, 234; GPR 2007, 222 (Klötgen); GPR 2007, 16 (Mansuy); GPR 2006, 174 (Klötgen); GPR 2006, 115 (Klötgen); GPR 2006, 19 (Mansuy/Schmied); GPR 2005, 172 (Mansuy); GPR 2005, 114; GPR 2005, 65 (Klötgen/Mansuy/ Cachard); GPR 2003/04, 250 (Klötgen/Mansuy/Cachard); GPR 2003/04, 177; GPR 2003/04, 122 (Klötgen/Cachard); GPR 2003/04, 72 (Klötgen/Mansuy/Cachard/Lambert)

- Länderberichte Italien von Omodei Salè, GPR 2020, 271 (Omodei Salè/Gatti); GPR 2020, 176 (Omodei Salè/Gatti); GPR 2020, 10 (Omodei Salè/Gatti); GPR 2019, 162 (Omodei Salè/Gatti); GPR 2019, 51 (Omodei Salè/Gatti); GPR 2018, 183; GPR 2018, 61; GPR 2017, 163; GPR 2017, 81; GPR 2016, 164; GPR 2016, 21; GPR 2015, 219; GPR 2014, 307; GPR 2013, 316; GPR 2013, 139; GPR 2012, 308; GPR 2011, 287; GPR 2011, 121; GPR 2010, 277; GPR 2010, 146; GPR 2008, 11; GPR 2007, 112; GPR 2006, 69; GPR 2005, 115; GPR 2003/04, 251; GPR 2003/04, 175; GPR 2003/04, 73 
- Länderberichte Österreich von Faber, GPR 2019, 215; GPR 2018, 180

- Länderberichte Polen von Tereszkiewicz, GPR 2011, 284 (Tereszkiewicz/Bobrzyński); GPR 2010, 225

- Länderberichte Spanien von Gascón Inchausti, GPR 2009, 74 (Martínez Santos); GPR 2008, 10 (Gascón Inchausti); GPR 2007, 168 (Gascón Inchausti); GPR 2007, 15 (Gascón Inchausti); GPR 2006, 17 (Gascón Inchausti); GPR 2005, 117 (Gascón Inchausti); GPR 2005, 16

- Länderberichte Tschechien von Semelová, GPR 2017, 165; GPR 2016, 166; GPR 2015, 173; GPR 2014, 136; GPR 2013, 85; von Navrátilová, GPR 2012, 64; GPR 2011, 74; GPR 2010, 70

- Länderberichte Ungarn von Harsági, GPR 2020, 210; GPR 2019, 218; GPR 2018, 216; GPR 2017, 227; GPR 2016, 220; GPR 2015, 221; GPR 2014, 209; GPR 2013, 203; GPR 2012, 189; GPR 2011, 166; GPR 2010, 170.

\section{b) Zum Europäischen IPR}

\section{aa) In der ZEuP}

Martiny, Europäisches Internationales Schuldrecht - Feinarbeit an Rom I- und Rom II-Verordnungen, ZEuP 2018, 218; Martiny, Europäisches Internationales Schuldrecht - Rom I- und Rom II-Verordnungen in der Bewährung, ZEuP 2015, 838; Martiny, Europäisches Internationales Schuldrecht Kampf um Kohärenz und Weiterentwicklung, ZEuP 2013, 838; Martiny, Neuanfang im Europäischen Internationalen Vertragsrecht mit der Rom I-Verordnung, ZEuP 2010, 747; Martiny, Europäisches Internationales Vertragsrecht in Erwartung der Rom I-Verordnung, ZEuP 2008, 79; Martiny, Neue Impulse im Europäischen Internationalen Vertragsrecht, ZEuP 2006, 60; Martiny, Europäisches Internationales Vertragsrecht vor der Reform, ZEuP 2003, 590; Martiny, Internationales Vertragsrecht im Schatten des Europäischen Gemeinschaftsrecht, ZEuP 2001, 308; Martiny, Europäisches Internationales Vertragsrecht - Ausbau und Konsolidierung, ZEuP 1999, 246; Martiny, Europäisches Internationales Vertragsrecht - Erosion der Römischen Konvention?, ZEuP 1997, 107; Martiny, Internationales Vertragsrecht zwischen Rechtsgefälle und Vereinheitlichung. Zum Römischen Übereinkommen vom 19. Juni 1980, ZEuP 1995, 67.

\section{bb) In der IPRax}

Mansel/Thorn/Wagner, Europäisches Kollisionsrecht 2019: Konsolidierung und Multilateralisierung, IPRax 2020, 97; Mansel/Thorn/Wagner, Europäisches Kollisionsrecht 2018: Endspurt!, IPRax 2019, 85; Mansel/Thorn/Wagner, Europäisches Kollisionsrecht 2017: Morgenstunde der Staatsverträge?, IPRax 2018, 121; Mansel/Thorn/Wagner, Europäisches Kollisionsrecht 2016: Brexit ante portas!, IPRax 2017, 1; Mansel/Thorn/Wagner, Europäisches Kollisionsrecht 2015: Neubesinnung, IPRax 2016, 1; Mansel/Thorn/Wagner, Europäisches Kollisionsrecht 2014: Jahr des Umbruchs, IPRax 2015, 1; Mansel/Thorn/Wagner, Europäisches Kollisionsrecht 2013: Atempause im status quo, IPRax 2014, 1; Mansel/Thorn/Wagner, Europäisches Kollisionsrecht 2012: Voranschreiten des Kodifikationsprozesses - Flickenteppich des Einheitsrechts, IPRax 2013, 1; Mansel/Thorn/Wagner, Europäisches Kollisionsrecht 2011: Gegenläufige Entwicklungen, IPRax 2012, 1; Mansel/Thorn/Wagner, Europäisches Kollisionsrecht 2010: Verstärkte Zusammenarbeit als Motor der Vereinheitlichung?, IPRax 2011, 1; Mansel/Thorn/Wagner, Europäisches Kollisionsrecht 2009: Hoffnungen durch den Vertrag von Lissabon, IPRax 2010, 1; Mansel/Thorn/Wagner, Europäisches Kollisionsrecht 2008: Fundamente der Europäischen IPR-Kodifikation, IPRax 2009, 1; Jayme/Kohler, Europäisches Kollisionsrecht 2007: Windstille im Erntefeld der Integration, 
IPRax 2007, 493; Jayme/Kohler, Europäisches Kollisionsrecht 2006: Eurozentrismus ohne Kodifikationsidee?, IPRax 2006, 573; Jayme/Kohler, Europäisches Kollisionsrecht 2005: Hegemonialgesten auf dem Weg zu einer Gesamtvereinheitlichung, IPRax 2005, 481; Jayme/Kohler, Europäisches Kollisionsrecht 2004:Territoriale Erweiterung und methodische Rückgriffe, IPRax 2004, 481; Jayme/Kohler, Europäisches Kollisionsrecht 2003 - Der Verfassungskonvent und das Internationale Privat- und Verfahrensrecht, IPRax 2003, 485; Jayme/Kohler, Europäisches Kollisionsrecht 2002: Zur Wiederkehr des Internationalen Privatrechts, IPRax 2002, 461; Jayme/Kohler, Europäisches Kollisionsrecht 2001: Anerkennungsprinzip statt IPR?, IPRax 2001, 501; Jayme/ Kohler, Europäisches Kollisionsrecht 2000: Interlokales Privatrecht oder universelles Gemeinschaftsrecht?, IPRax 2000, 454; Jayme/Kohler, Europäisches Kollisionsrecht 1999 - Die Abendstunde der Staatsverträge, IPRax 1999, 401; Jayme/Kohler, Europäisches Kollisionsrecht 1998: Kulturelle Unterschiede und Parallelaktionen, IPRax 1998, 417; Jayme/Kohler, Europäisches Kollisionsrecht 1997 - Vergemeinschaftung durch „Säulenwechsel“?, IPRax 1997, 385; Jayme/Kohler, Europäisches Kollisionsrecht 1996 - Anpassung und Transformation der nationalen Rechte, IPRax 1996, 377; Jayme/Kohler, Europäisches Kollisionsrecht 1995 - Der Dialog der Quellen, IPRax 1995, 343; Jayme/Kohler, Europäisches Kollisionsrecht 1994 - Quellenpluralismus und offene Kontraste, IPRax 1994, 405; Jayme/Kohler, Das Internationale Privat- und Verfahrensrecht der EG 1993 - Spannungen zwischen Staatsverträgen und Richtlinien, IPRax 1993, 357; Jayme/ Kohler, Das Internationale Privat- und Verfahrensrecht der EG nach Maastricht, IPRax 1992, 346; Jayme/Kohler, Das Internationale Privat- und Verfahrensrecht der EG 1991 - Harmonisierungsmodell oder Mehrspurigkeit des Kollisionsrechts, IPRax 1991, 361; Jayme/Kohler, Das Internationale Privat- und Verfahrensrecht der EG auf dem Wege zum Binnenmarkt, IPRax 1990, 353; Jayme/Kohler, Das Internationale Privat- und Verfahrensrecht der EG - Stand 1989, IPRax 1989, 337; Jayme/Kohler, Das Internationale Privat- und Verfahrensrecht der Europäischen Gemeinschaft - Jüngste Entwicklungen, IPRax 1988, 133; Jayme/Kohler, Zum Stand des internationalen Privat- und Verfahrensrechts der Europäischen Gemeinschaft, IPRax 1985, 65.

\section{cc) In der GPR}

Arnold/Zwirlein-Forschner, Die Entwicklung der Rechtsprechung zum Europäischen Internationalen Privatrecht, GPR 2019, 262; Arnold/Zwirlein, Die Entwicklung der Rechtsprechung zum Internationalen Privatrecht, GPR 2018, 221; Arnold, Die Entwicklung der Rechtsprechung zum Internationalen Privatrecht, GPR 2017, 29; Pabst, Entwicklungen im europäischen und völkervertraglichen Kollisionsrecht 2011-2012, GPR 2013, 171; Rauscher/Pabst, Entwicklungen im europäischen und völkervertraglichen Kollisionsrecht 2009-2010, GPR 2011, 41; Rauscher/Pabst, Entwicklungen im europäischen und völkervertraglichen Kollisionsrecht 2008-2009, GPR 2009, 294; Rauscher/Pabst, Entwicklungen im europäischen und völkervertraglichen Kollisionsrecht 20072008, GPR 2008, 302; Rauscher/Pabst, Entwicklungen im europäischen und völkervertraglichen Kollisionsrecht 2005-2007, GPR 2007, 244.

\section{dd) In der NJW}

Rauscher, Die Entwicklung des Internationalen Privatrechts 2019 bis 2020, NJW 2020, 3632; Rauscher, Die Entwicklung des Internationalen Privatrechts 2018 bis 2019, NJW 2019, 3486; Rauscher, Die Entwicklung des Internationalen Privatrechts 2017 bis 2018, NJW 2018, 3421; Rauscher, Die Entwicklung des Internationalen Privatrechts 2016 bis 2017, NJW 2017, 3486; Rauscher, Die Entwicklung des Internationalen Privatrechts 2015 bis 2016, NJW 2016, 3493; Rauscher, Die 
Entwicklung des internationalen Privatrechts 2014 bis 2015, NJW 2015, 3551; Rauscher, Die Entwicklung des Internationalen Privatrechts 2013-2014, NJW 2014, 3619; Rauscher/Pabst, Die Entwicklung des Internationalen Privatrechts 2012-2013, NJW 2013, 3692; Rauscher/Pabst, Die Entwicklung des Internationalen Privatrechts 2011-2012, NJW 2012, 3490; Rauscher/Pabst, Die Rechtsprechung zum Internationalen Privatrecht 2010-2011, NJW 2011, 3547; Rauscher/Pabst, Die Rechtsprechung zum Internationalen Privatrecht 2009-2010, NJW 2010, 3487; Rauscher/ Pabst, Die Rechtsprechung zum Internationalen Privatrecht 2008-2009, NJW 2009, 3614; Rauscher/Pabst, Die Rechtsprechung zum Internationalen Privatrecht 2007-2008, NJW 2008, 3477; Rauscher/Pabst, Die Rechtsprechung zum Internationalen Privatrecht 2005-2007, NJW 2007, 3541.

\section{Quellen im Internet}

\section{a) EuGH-Urteile}

Sämtliche Entscheidungen des EuGH lassen sich auf der Seite des Gerichts recherchieren: http://curia.europa.eu/juris/recherche.jsf?language=de

\section{b) EU-Rechtsakte}

Eine Datenbank zu sämtlichen EU-Rechtsakten findet sich auf der Seite der EU-Kommission (auch Amtsblatt der EU, Verträge, Rechtsvorschriften): http://eur-lex.europa.eu/homepage.html Auch die Seite des Rates enthält die vom Europäischen Rat bzw. vom Rat der EU seit 1999 verfassten Dokumente: http://www.consilium.europa.eu/de/documents-publications/

\section{c) Gesetzgebungsverfahren}

Der Fortgang von Gesetzgebungsverfahren in der EU lässt sich im System EUR-Lex nachvollziehen: https://eur-lex.europa.eu/advanced-search-form.html?locale=de

Laufende Arbeiten der in den einzelnen Ausschüssen anhängigen Legislativverfahren des Europäischen Parlaments lassen sich hier nachverfolgen: http://www.europarl.europa.eu/committees/ de/juri/documents/work-in-progress.

\section{d) Sonstige Informationen}

Pressemeldungen der Europäischen Kommission - Vertretung in Deutschland: https://ec.europa.eu/ germany/news_de

Allgemeine Pressemeldungen der Europäischen Kommission: http://ec.europa.eu/newsroom/just/ news-overview.cfm 
